\title{
A Moving Average based Filtering System with its Application to Real-time QRS Detection
}

\author{
HC Chen, SW Chen
}

\author{
Department of Electronic Engineering, Chang Gung University, Taiwan
}

\begin{abstract}
This paper presents a novel real-time QRS detection algorithm designed based on a simple moving average filter. The proposed algorithm demands no redundant preprocessing step, thus allowing a simple architecture for its implementation as well as low computational cost. Algorithm performance was validated against a subset of the MIT-BIH Arrhythmia Database. Consequently, numerical results showed that the proposed algorithm correctly detected over $99.5 \%$ of the QRS complexes from the standard ECG database, implying it may be considered as a simple and reliable candidate of $Q R S$ detection algorithms.
\end{abstract}

\section{Introduction}

QRS detection serves as the fundamentals for a wide variety of automated cardiac signal analysis algorithms [1]. According to previous researches in literature, a number of algorithms have been developed to detect the QRS complex from the electrocardiogram (ECG) [1]-[7]. Since the shape of QRS complex is time varying, and is subject to physiological variations as well as to corruption due to noise [2], a reliable QRS detection algorithm is thus essentially demanded in many aspects of applications into the ECG analysis.

Some existing QRS detection algorithms employ a specific QRS template [5]-[7], which might be considered the best way to prevent the QRS detection performance from being degraded by the undesired noise sources, including baseline drifts, artifacts due to electrode motion or power-line interference, and the waves with similar morphology to QRS complexes, such as P or T waves [3]. However, since the template technique involves intensive cross correlation-based similarity measurement between the QRS template and a number of windowed ECG signals, such a heavy computational burden somehow might undesirably restrict its use to quite a limited number of aspects [8]. In this paper, a simple and reliable moving averaging-based real-time QRS detection algorithm is proposed. The novel algorithm demands no extra digital filters for preprocessing step, permitting a reduction in the computational cost and simple realization.

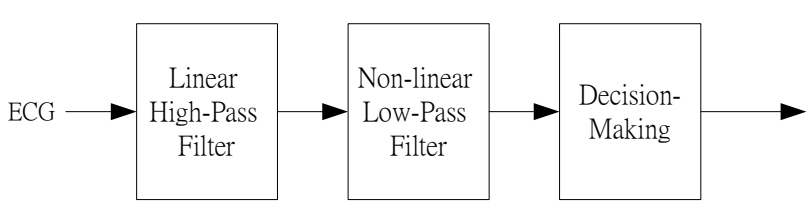

Figure 1. A block diagram of the QRS detection system.

\section{Methodology}

Fig. 1 gives a schematic block diagram of the proposed QRS detection system. In general, the overall detection system is mainly composed of three processing stages: a moving average-based highpass filtering (HPF), a nonlinear lowpass filtering (LPF), and a decisionmaking stage. The task of QRS detection is performed as follows. First, an ECG recording is processed by the linear HPF in order to accentuate the QRS complex, and meanwhile, to suppress the undesired waves of ECG, such as $\mathrm{P}$ or $\mathrm{T}$ waves, as well as the baseline wander. After that, in order to guarantee that the high-frequency, low-amplitude artifacts can be smoothed down to a certain level while the QRS feature can be well preserved, the linear HPF output is then processed by a full-wave rectification and nonlinear amplification followed by a sliding-window summation, thus resulting in an envelope-like feature waveform. All the operations addressed above can be attributed to a nonlinear LPF process. Finally, an adaptive threshold is applied to the feature waveform to perform the task of decision-making for completing a QRS complex detection. Details are presented in the following subsections.

\subsection{Linear High-Pass Filtering Stage}

At this stage, a linear filtering system is composed of an $M$-point moving average filter (MAF) and an ideal delayed system with a group delay of $(M+1) / 2$ samples with both processing elements connected in a parallel form, as indicated in Fig. 2. For convenience, we restrict $M$ to odd values. The MAF output is then subtracted, point-by-point, from the delayed input sample so the entire system becomes an FIR HPF with linear phase. 


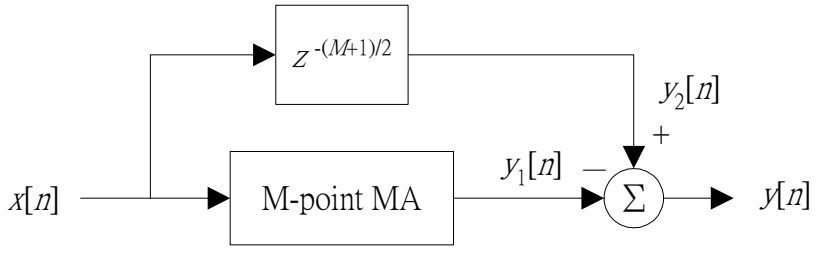

Figure 2. The architecture of the HPF

Observing Fig. 2, first the input-output relation of a causal MAF can be characterized as

$$
y_{1}[n]=\frac{1}{M} \sum_{m=0}^{M-1} x[n-m]
$$

where $x[n]$ represents the input data and $M$ is the filter length. Meanwhile, it can be seen from Fig. 2 that the input signal $x[n]$ is also simultaneously passed through an ideal delayed system with a group delay of $(M+1) / 2$ samples, resulting in

$$
y_{2}[n]=x\left[n-\frac{M+1}{2}\right]
$$

According to Fig. 2, the overall system output $y[n]$ is determined simply by subtracting $y_{1}[n]$ from $y_{2}[\mathrm{n}]$, that is,

$$
\begin{aligned}
y[n] & =y_{2}[n]-y_{1}[n] \\
& =x\left[n-\frac{M+1}{2}\right]-\frac{1}{M} \sum_{m=0}^{M-1} x[n-m]
\end{aligned}
$$

Eq. (3) then algebraically results in

$$
\begin{aligned}
y[n]= & -\frac{1}{M} x[n]-\cdots-\frac{1}{M} x\left[n-\frac{M-1}{2}\right]+\frac{M-1}{M} x\left[n-\frac{M+1}{2}\right] \\
& -\frac{1}{M} x\left[n-\frac{M+3}{2}\right]-\cdots-\frac{1}{M} x[n-M+1]
\end{aligned}
$$

Note that Eq. (4) is valid only if the filter length $M$ is restricted to odd values. As a result, the overall system depicted in Fig. 2 becomes a type I FIR HPF with linear phase. In fact, for the purpose of a linear phase HPF realization, $M$ is not allowed to be even values. This is because even values of $M$ will result in a type II linear phase FIR filter and it is highly unsatisfactory [9], due to the zero of $H(z)$ that is forced by the linear phase constraint to be $z=-1$, i.e., $\omega=\pi$, indicating there always exists a zero at the highest frequency and thus not suitable for linear phase HPF design.

In general, the HPF stage employed here is intended to emphasize the QRS complex while suppressing the lower frequency noise sources of an ECG signal such as $\mathrm{P}$ or $\mathrm{T}$ waves, as well as the baseline wander. Therefore, selection of $M$ is also an important issue. Different values of $M$ will result in different frequency responses of

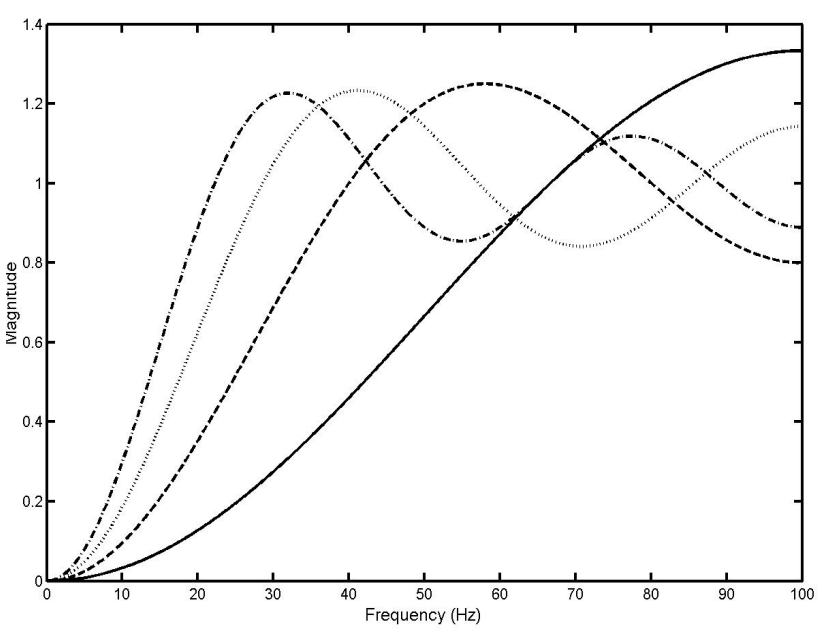

Figure 3. Frequency responses of the MAF's with various filter lengths (solid: 3-point MA, dash: 5-point MA, dot: 7-point MA, dash-dot: 9-point MA)

the HPF, as manifested in Fig. 3, and consequently might lead to different QRS detection performances. Considering the relative power spectra of QRS complex and P-T waves, the energy associated with the former mainly concentrates over approximately $5-15 \mathrm{~Hz}$ while a majority of energy associated with the latter or baseline wander typically distributes over the frequencies less than $5 \mathrm{~Hz}$. Observing Fig. 3 it can be seen that a smaller $M$ (say, $M=3$ ) might not adequately emphasize the QRS complex in the ECG signal, in comparison with other cases. On the other hand, the low frequency noise sources such as $\mathrm{P}$ or $\mathrm{T}$ waves will be undesirably enhanced if $M$ is too big (say, $M=9$ ). In this study, we therefore suggest an appropriate selection of $M$ value can be $M=5$ or 7 .

\subsection{Nonlinear Low-Pass Filtering Stage}

The output of the HPF is further inputted into a nonlinear LPF constructed by a cascade of a simple point-by-point squaring operation and a moving window integration or summation system, as depicted in Fig. 4.

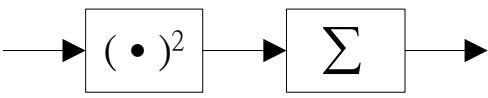

Figure 4. The component of nonlinear LPF

As mentioned earlier in Section 2, the nonlinear LPF can be alternatively viewed as a nonlinear envelope detector, since it producing envelope-like feature waveforms. Moreover, the width of the moving summation window is also important. Generally speaking, the width should be appropriately selected so the summation waveform will neither result from a merge of the QRS and T waves together nor be a number of peaks 
produced by a single QRS complex. In fact, this width should be determined by taking the sampling rate of the original ECG signal into consideration. A previous study regarding this issue suggested that, empirically, an appropriate choice of this summation interval could be 30 samples wide as the ECG sampling rate is $200 \mathrm{~Hz}$, corresponding to $150 \mathrm{~ms}$ in real-time [3], [10].

Consequently, with appropriate choice of $M$ the peak level of the moving window summation of the squared linear HPF output corresponding to a QRS complex appeared to be significantly enhanced while those corresponding to the undesired noise peaks, such as the $\mathrm{P}$ and $\mathrm{T}$ waves were relatively attenuated, as illustrated in the lower panel of Fig. 5. In fact, observations gathered from the above allow us to devise a simple scheme of thresholding for the final decision-making stage.

\subsection{Decision-Making Stage}

Here, an adaptive threshold is incorporated into the scheme designed for decision-making and is updated by

$$
\text { Threshold }=\alpha \times \gamma \times P E A K+(1-\alpha) \times \text { Threshold }
$$

where $P E A K$ is the local maximum newly detected in the feature waveform and $\alpha$ is referred to as the "forgetting factor," restricted to the positive fraction numbers, that is, $0 \leqq \alpha \leqq 1$. According to Eq. (5) it can be seen that each new value of the threshold is determined from the running estimate of the feature signal peak as well as the prior and current values of the threshold itself. $\gamma$ is a weighting factor for determining the contribution of peak values to threshold adjustment. Empirically, the values of $\gamma$ can be 0.15 or 0.2 . A QRS complex is said to be detected, only if the peak level of the feature signal exceeds the threshold. The value of the threshold is then updated each time when a new QRS complex is detected. A demonstrative example of the QRS detection addressed above is also given in Fig. 5.

\section{Results}

The novel algorithm was tested and validated against a subset of the MIT-BIH Arrhythmia Database. The optimal numerical experimental results for the subset of this standard database are summarized in Table 1 . We may see from the table that the proposed algorithm produced 113 false positives (FP's) and 160 false negatives (FN's), resulting in a total correct detection rate over $99.5 \%$. Note that here the forgetting factor $\alpha$ was not restricted to a certain fixed value (say, 0.05). Instead, according to our numerical experiments $\alpha$ can be chosen over a certain range approximately from 0.1 to 0.01 . Moreover, it was also found that the error detection rates were all less than $1 \%$ with $M=3,5,7$. Although the results obtained from some recordings were not as good
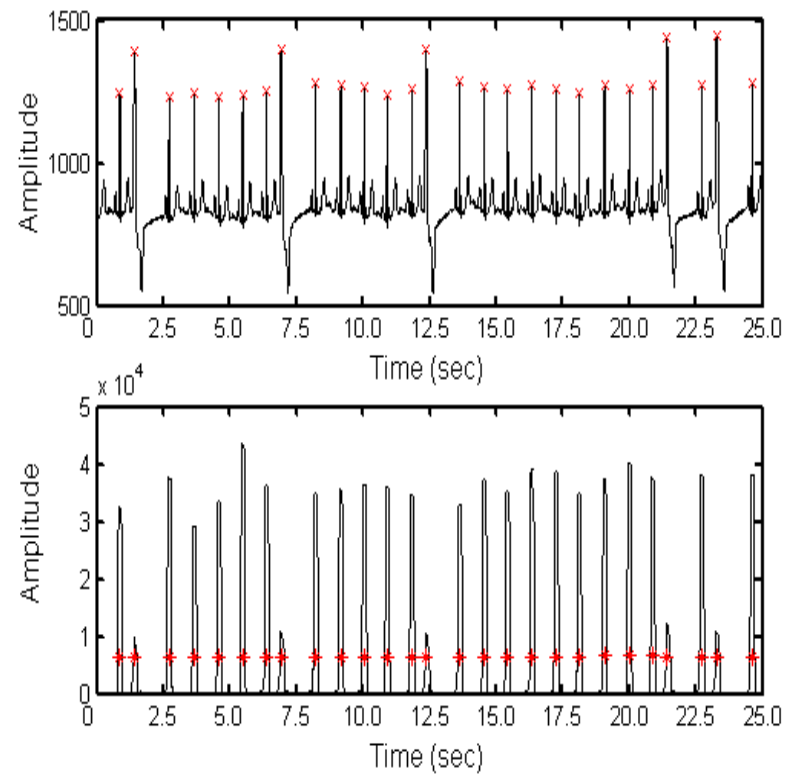

Figure 5. The upper plot is the original ECG signal, and the symbol " $x$ " indicates the detected QRS complex. The lower plot gives the feature signal obtained after the nonlinear LPF process, and the symbol "*” indicates the adaptive threshold value.

Table 1. Results of performance evaluation for the proposed real-time QRS detection algorithm with $M=5$, $\alpha=0.05 \gamma=0.15$

\begin{tabular}{cccrrr}
\hline \hline $\begin{array}{c}\text { Tape } \\
\text { (No.) }\end{array}$ & $\begin{array}{c}\text { Total } \\
\text { (No. Beats) }\end{array}$ & $\begin{array}{c}\text { FP } \\
\text { (Beats) }\end{array}$ & $\begin{array}{c}\text { FN } \\
\text { (Beats) }\end{array}$ & $\begin{array}{c}\text { Failed } \\
\text { Detection } \\
\text { (Beats) }\end{array}$ & $\begin{array}{c}\text { Failed } \\
\text { Detection } \\
(\%)\end{array}$ \\
\hline 100 & 2273 & 1 & 0 & 1 & 0.04 \\
101 & 1865 & 7 & 3 & 10 & 0.54 \\
103 & 2084 & 0 & 0 & 0 & 0.00 \\
109 & 2532 & 7 & 24 & 31 & 1.22 \\
113 & 1795 & 0 & 0 & 0 & 0.00 \\
114 & 1879 & 13 & 4 & 17 & 0.90 \\
115 & 1953 & 0 & 0 & 0 & 0.00 \\
116 & 2412 & 5 & 20 & 25 & 1.04 \\
117 & 1535 & 3 & 2 & 5 & 0.33 \\
119 & 1987 & 0 & 0 & 0 & 0.00 \\
122 & 2476 & 0 & 0 & 0 & 0.00 \\
123 & 1518 & 2 & 3 & 5 & 0.33 \\
124 & 1619 & 1 & 0 & 1 & 0.06 \\
201 & 1963 & 2 & 13 & 15 & 0.76 \\
202 & 2136 & 8 & 7 & 15 & 0.70 \\
205 & 2656 & 1 & 9 & 10 & 0.38 \\
209 & 3004 & 28 & 8 & 36 & 1.20 \\
213 & 3251 & 0 & 26 & 26 & 0.80 \\
219 & 2154 & 0 & 3 & 3 & 0.14 \\
220 & 2048 & 0 & 0 & 0 & 0.00 \\
221 & 2427 & 9 & 19 & 28 & 1.15 \\
222 & 2483 & 16 & 8 & 24 & 0.97 \\
230 & 2256 & 8 & 0 & 8 & 0.35 \\
231 & 1571 & 0 & 6 & 6 & 0.38 \\
234 & 2753 & 2 & 5 & 7 & 0.25 \\
\hline 25 patients & 54630 & 113 & 160 & 273 & 0.46
\end{tabular}


as those from others, possibly due to severe noise contaminations (particularly the sudden change of the signal quality, for example), all the results of performance evaluation presented above still manifested a degree of flexibility and robustness for the proposed algorithm.

\section{Conclusion}

In this paper, a real-time moving averaging-based QRS detection algorithm is introduced. The novel algorithm can reliably detect QRS complex. According to our study, it appeared while achieving a pretty high QRS detection rate, the proposed algorithm requires no redundant preprocessing filters thus permitting relative fewer calculations, in comparison with most existing QRS detection algorithms. In addition, it was also found from our study that there existed a degree of flexibility for parameter value selection as well as of robustness over a wide range of noise contamination.

\section{Acknowledgements}

This work was supported by the National Science Council, Taiwan, under Contract NSC-91-2218-E-182-002.

\section{References}

[1] Köhler BU, Hennig C, Orglmeister R. The principles of software QRS detection. IEEE Eng Med Biol Mag 2002;21:42-57.
[2] Friesen GM, Jannett TC, Jadallah MA, Yates SL, Quint, SR, Nagle HT. A comparison of the noise sensitivity of nine QRS detection algorithms. IEEE Trans Biomed Eng 1990;37:85-98

[3] Pan J, Tompkins WJ. A real-time QRS detection algorithm. IEEE Trans Biomed Eng 1985;32:230-236.

[4] Suppappola S, Sun Y. Nonlinear transform of ECG signals for digital QRS detection: a quantitative analysis. IEEE Trans Biomed Eng 1994;41:397-400.

[5] Cohen A, Landsberg D. Adaptive real-time wavelet detection. IEEE Trans Biomed Eng 1983;30:332-340.

[6] Dobbs SE, Schmitt NM, Ozemek HS. QRS detection by template matching using real-time correlation on a microcomputer. J Clin Eng 1984;9:197-212.

[7] Xue Q, Hu YH, Tompkins WJ. Neural-network-based adaptive matched filtering for QRS detection. IEEE Trans Biomed Eng 1992;39:317-329.

[8] Bartolo A, Clymer BD, Burgess RC, Turnbull JP, Golish JA, Perry MC. An arrhythmia detector and heart rate estimator for overnight polysomnography studies. IEEE Trans Biomed Eng 2001;48:513-521.

[9] Oppenheim AV, Schafer RW. Discrete-time Signal Processing. Second edition. Prentice Hall, 1999.

[10] Tompkins WJ. Biomedical Digital Signal Processing. Prentice Hall, 1993.

Address for correspondence.

Szi-Wen Chen

Department of Electronic Engineering, Chang Gung University 259 Wen-Hwa $1^{\text {st }}$ Road, Kwei-Shan, Tao-Yuan, Taiwan tel: +886-3-2118800 ext.5792

chensw@mail.cgu.edu.tw 